\title{
Soft tissue facial changes among adult females during the first six months of orthodontic treatment: a spatially dense 3D geometric morphometric study
}

Si Yu Hou

Chongqing Medical University

\section{Wenwen Zhou}

Chongqing Medical University Stomatology College

Hongwei Dai

Chongqing Medical University

Hai Ming Wong

University of Hong Kong

Yi Feng Wen

college of stomatology, Xi'an Jiaotong University

Jianping Zhou ( $\nabla$ zjp@hospital.cqmu.edu.cn )

Chongqing Medical University

Research article

Keywords: soft tissue, face, orthodontic treatment, geometric morphometrics, quasi-landmarks

Posted Date: September 2nd, 2020

DOl: https://doi.org/10.21203/rs.3.rs-35933/v2

License: (1) This work is licensed under a Creative Commons Attribution 4.0 International License.

Read Full License 


\section{Abstract}

Background: To investigate changes in facial morphology during the first six months of orthodontic treatment among adult females receiving orthodontic treatment.

Methods: 18 adult females receiving orthodontic treatment were randomly recruited. 3D facial images were taken at baseline (T0), three months (T1), and six months (T2) after treatment initiation. Spatially dense facial landmarks were digitized to allow for sufficient details in characterization of facial features. $3 \mathrm{D}$ geometric morphometrics and multivariate statistics were used to investigate changes in mean and variance of facial shape and facial form associated with treatment.

Results: We observed statistically significant changes in facial shape across the three treatment stages ( $p$ $=0.003)$. Pairwise comparisons suggested significant changes from T0 to T1 $(p=0.0059)$ and from T0 to $T 2(p=0.0098)$. Heatmap visualization indicated that the buccal and temporal region were invaginated while the labial region became protruded with treatment. The magnitude of shape change was 0.009 , 0.004, and 0.010 from T0 to T1, T1 to T2, and T0 to T2, respectively, in unit of Procrustes distance. The average magnitude of change per-landmark was $1.31 \mathrm{~mm}, 0.22 \mathrm{~mm}$, and $1.33 \mathrm{~mm}$, respectively. Changes in mean facial form were not statistically significant $(p=0.103)$. No changes in variance of facial shape were observed across treatment stages $(p>0.05)$.

Conclusion: Rate of facial changes was twice as fast during the first three months as that during fourth to sixth month. Buccal and temporal region became invaginated while labial region became protruded with treatment.

\section{Background}

The importance of a harmonious and aesthetic facial appearance among women is deeply rooted evolutionarily and societally. The number of adult females seeking orthodontic treatment has been on a rise (1). Improving self-image accounted for more than $70 \%$ of adult orthodontic patients' reasons for seeking orthodontic treatment $(2,3)$.

Studies on soft tissue facial changes of adult orthodontic patients revealed that the face of patients who received tooth extraction became flattened during treatment while it became fuller among non-extraction patients $(4,5)$. It should be noted that these studies were performed based on facial profile of 2D images. Therefore, only facial changes in the midsagittal plane could be evaluated (6). Taking advantage of 3D facial imaging technology, Moss et al. observed complex patterns of facial changes with treatment among adolescent orthodontic patients (7). In awareness of the complexity of facial structures, Kab et al. divided 3D facial surface into multiple regions for separate analysis (8).

Previous studies of adult facial changes during orthodontic treatment mostly focused on pre- and posttreatment changes of the labial region based on cephalograms. There is currently a lack of understanding of the dynamics of facial changes that take place during the treatment process. In addition, facial 
changes other than the labial region remain elusive. Qin et al. (9) suggested that orthodontic treatment among adults is likely to result in "bracket face" within 1-2 months after treatment, characterized by invaginated cheeks and more prominent zygomatic region which collectively renders a greater-thanactual-age facial appearance. However, without a thorough understanding of facial changes associated with orthodontic treatment, the notion of "bracket face" can hardly be universally accepted.

Conventional facial morphometrics have heavily relied on traditional linear, angular, and proportion measurements (10). While these measurements are straightforward to understand, each measurement provides only very limited morphometric information. Increasing the number of facial measurements, unfortunately, would make interpretation of key facial changes challenging $(11,12)$. Geometric morphometrics (GM) is a revolutionary quantitative morphometric approach based on rigorous statistical theory of shape (13). Instead of analyzing each facial measurement individually, GM retains the shape and form (shape + size) information encoded by all landmarks during analysis (14). This facilitates powerful multivariate statistical analysis of facial shape/form and allows for direct visualization of facial shape/form differences.

The use of GM in soft tissue facial analysis is emerging in recent years. Wen et al. (15) investigated facial shape development among Hong Kong adolescents aged 12 to 18 years based on frontal and lateral facial photographs. In the field of orthodontics, Kouli et al. (6) applied GM to investigate changes in facial profile before and after orthodontic treatment. Most current studies are based on 2D images, which are inherently limited in providing a comprehensive understanding of facial changes in 3D. Abedini et al. (16) examined 3D facial changes following micro-implant-supported maxillary skeletal expansion based on facial stereophotogrammetry. However, this study has several methodological limitations. First, the authors' approach to create left and right expanded group cannot adequately extract the symmetric component of facial shape. As is common in GM, the average of each original configuration and its relabeled reflection (mirrored and labels of bilateral landmarks switched to ensure original left- and rightside landmarks were respectively averaged with reflected right- and left-side landmarks) gives the symmetric component of the configuration (17). Second, statistical evaluation of treatment-related facial changes was performed without regard to the repeated-measures design of the study, which reduced its power to identify facial regions with statistically significant changes. Third, the exact number of facial landmarks used for analysis was not reported. GM based on spatially dense facial quasi-landmarks (landmarks without distinct anatomical definitions) and the use of appropriate multivariate statistics taking repeated-measures design into consideration are clearly warranted to gain in-depth, high-resolution understanding of natural and treatment-related facial changes.

The present study aimed to apply GM and multivariate statistics to evaluate changes of facial shape and form from baseline through 3 months to 6 months of orthodontic treatment among adult female patients.

\section{Methods}

\section{Study sample}


This study was approved by the Research Ethics Board of the Stomatological Hospital of Chongqing Medical University (No. 2020-013). All patients gave informed consent prior to participation. Patients for this study were recruited from consecutive adult patients visiting the Department of Orthodontics, Stomatological Hospital of Chongqing Medical University, Chongqing, China. A total of 18 females aged 18-26 years were recruited for this study. The mean age of the patients were 22.6 years. Of the 18 patients, 15 received extraction of four first or second premolars prior to the start of orthodontic treatment while 3 patients received orthodontic treatment without tooth extraction.

Patients eligible for this study should satisfy all of the following criteria: adult females of Chinese ethnicity, ANB angle between 0 to 4 degrees, and Body Mass Index within the range of 18.5 to 25 , which represented individuals of normal weight (18). Patients with obvious facial asymmetry, craniofacial anomalies, previous history of orthodontic treatment, and defective dentitions were excluded.

All patients were treated with the same fixed appliances $(0.022 \times 0.028$-inch bracket slot). Mandibular bracket was bonded one month after treatment began. The order in which nickel-titanium archwire sequence used were 0.012 inch, 0.014 inch, 0.016 inch, 0.0160 .022 inch, and 0.0180 .025 inch.

\section{Facial surface imaging}

Digital facial stereophotogrammetry (Morpheus 3D, Korea) was used to capture 3D facial surfaces for each individual. Patients were imaged following standard facial image acquisition protocol (19). Patients were asked to gently close mouth, maintain neutral facial expression, and assume natural head position during imaging. Images were taken at three treatment stages: baseline (T0), three months after treatment initiation (T1), and six months after treatment initiation (T2).

\section{Spatially dense facial quasi-landmarking}

3D facial images obtained from the Morpheus 3D systems was stored in .M3D format, which was converted to the OBJ format by the company. Facial images in OBJ format was further converted to ASCII PLY format. Each facial image in PLY format was imported into the IDAV Landmark Editor v.3.0.0.6 to digitize five anchoring points (right exocanthus, left exocanthus, pronasale, right cheilion, left cheilion) in a fixed order (Figure 1A) (20).

Facial images in $\mathrm{OBJ}$ format, together with coordinates of the five anchoring points, were imported into the MeshMonk toolbox of MATLAB (R2018b) for spatially dense facial quasi-landmarking (21). Based on the five anchoring points, an anthropometric mask was mapped to each facial image through rigid and non-rigid registration algorithms (Figure $1 \mathrm{~B}-\mathrm{C}$ ). This resulted in 7160 3D facial quasi-landmarks that capture facial region of interest while removing irrelevant structures such as hair, ears, and any dissociated polygons (22).

\section{Generalized Procrustes Analysis}


Human face is internally symmetric around the midsagittal plane (23). Each quasi-landmark on the right side had a homologous quasi-landmark on the left side. We reflected and relabeled the quasi-landmark configuration of each patient's face. All configurations and their relabeled reflections were superimposed through Generalized Procrustes Analysis (GPA). This removed among-configuration variation in size, location, and orientation (24) and resulted in Procrustes shape coordinates that characterized facial shape (25).

Although orthodontic treatment may impact left and right side of the face differentially, this is not the focus of the present study. Therefore, all GM facial analysis in this study was performed based on the symmetric component of facial shape.

\section{Changes in mean facial shape across treatment stages}

Statistical significance of changes of facial shape from T0 through T1 to T2 was evaluated using permutational multivariate analysis of variance (MANOVA) of distance matrices comprising pairwise Procrustes distance (PD) between configurations (26). Procrustes shape coordinates were used as response variable and treatment stage, treatment modality (tooth extraction vs non-extraction), and their interactions were used as explanatory variables. Unlike traditional MANOVA in which groups under comparison were independent, facial shape at T0, T1, and T2 were correlated because they represented repeated measurements on the same group of patients. Therefore, we constrained permutation to be within each participant, as recommended by Anderson and Braak (2003), to preclude the confounding effect of among-patient variation on evaluations of facial shape changes during treatment (13). Empirical $p$ values were calculated as the probability that the permuted pseudo F-statistic was larger or equal to the observed F-statistic (26), based on 10000 permutations. In addition, coefficient of partial determination ( was calculated for each explanatory variable following the formula by Kleinbaum et al. (27).

To gain an understanding of facial regions where shape changed significantly with treatment, we determined statistical significance and relative magnitude of positional changes of each facial quasilandmark. The analyses were performed based on the same model used above while replacing Procrustes shape coordinates for the entire face with 3D coordinates for each facial quasi-landmark. Similar approach has been adopted by Zaidi et al. (28) and Claes et al. (29).

To ascertain the specific treatment stages between which facial shape changed significantly, post-hoc pairwise comparisons were performed. The magnitude of shape change was quantified by PD between mean facial shape of the two treatment stages under comparison. The permutational MANOVA was performed using the Adonis function of the vegan package version 2.5-6 in R version 4.0.0 (30,31). 3D Facial heatmaps were used for visualization of study findings.

\section{Changes in variance of facial shape across treatment stages}


Variance of the facial shape was estimated as Procrustes variance (32). The morphol.disparity function in geomorph package version 3.2.1 in $\mathrm{R}$ was used to quantify pairwise differences in variance of facial shape among all treatment stage-by-modality groups $(29,33)$.

In additional to the above analyses of facial shape, facial form was obtained by multiplying Procrustes shape coordinates of each facial image with its corresponding centroid size. Statistical significance of changes in mean and variance of facial form was performed following the same procedures described above. The level of statistical significance was set at 0.05 for all analyses. Quasi-landmark repeatability error of MeshMonk has been reported to be as low as 0.002 in unit of Procrustes distance, which is robust to manual landmark digitization errors associated with digitization of the five anchoring points (34).

\section{Results}

\section{Changes in mean facial shape across treatment stages}

Permutational MANOVA suggested that changes in mean facial shape were statistically significant across treatment stages $(p=0.003)$. Pairwise comparisons suggested that facial shape changed significantly from T0 to T2 $(p=0.0098)$. Figure $2 A$ revealed invaginated buccal and temporal region in contrast to protruding labial region with treatment. Pairwise comparison also revealed significant facial changes from T0 to T1 ( $p=0.0059)$. Facial changes from T0 to T1 (Figure 2B) were indistinguishable from changes during T0 to T2 (Figure 2A). Changes of facial shape from T1 to T2 were characterized by widespread retraction of the mid- and lower-facial third with concomitant perioral protrusion. However, these changes were not statistically significant $(p=0.4728)$. The magnitude of changes in facial shape was 0.009 from T0 to T1, 0.004 from T1 to T2, and 0.010 from T0 to T2 in units of Procrustes distance.

Distribution of magnitude of changes of facial landmarks was illustrated in Figure 3. The magnitude of changes was $1.31 \mathrm{~mm}$ (SD: $0.39 \mathrm{~mm}$ ), $0.22 \mathrm{~mm}$ (SD: $0.11 \mathrm{~mm}$ ), and $1.33 \mathrm{~mm}$ (SD: $0.46 \mathrm{~mm}$ ) from T0 to $T 1, T 1$ to $T 2$, and $T 0$ to $T 2$, respectively. Quasi-landmarks whose positional changes explained the greatest amount of across-treatment stage facial shape changes were concentrated around buccal, temporal, and labial region. Per-landmark analysis revealed similar patterns of facial changes (Figure 4) compared to changes suggested by analysis of the entire set of Procrustes shape coordinates (Figure 2).

\section{Changes in variance of facial shape across treatment stages}

Changes in variance of facial shape was not statistically significant across treatment stages regardless of whether patients received tooth extraction prior to the start of orthodontic treatment (Table 3). Likewise, no changes were observed for variance of facial form across treatment stages in either treatment group (Table 3).

\section{Discussion}


To our knowledge, this is the first study that investigated longitudinal changes in facial shape/form among adult female orthodontic patients using GM approach based on spatially dense facial quasilandmarks. Our findings suggest buccal and temporal region invaginated and labial region protruded, in terms of facial shape, as a result of orthodontic treatment. These changes were concentrated within the first three months of treatment. No changes in facial form were observed. Variance of facial shape and form were found constant during the first six months of treatment.

Wirthlin et al. (35) reported an average yearly facial change of $0.15 \mathrm{~mm}$ among females from age 22 to 33 years. The average quasi-landmark change of $1.33 \mathrm{~mm}$ we observed within the first six months of treatment is a magnitude larger than the amount of natural changes reported. Similarly, Takoma et al. (36) found no significant changes in female facial morphology after age 18 years. Qin et al. (9) likewise indicated no significant facial changes among females from 20 to 30 years of age. Therefore, the facial changes we observed is unlikely to be an artefact of natural changes of facial shape that take place during the investigation period.

Both the entire set of facial Procrustes coordinates and per-landmark analysis converged in revealing invaginated buccal and temporal region and protruded labial region. The changes in buccal and temporal regions were likely associated with changes in occlusion and masticatory muscle during orthodontic treatment. As soon as nickel-titanium archwire is ligated, patients report strong pain and tend to avoid chewing (37). In addition, unstable occlusion during orthodontic treatment further decrease masticatory performance and impair chewing efficiency during initial stages of treatment $(38,39)$. Such changes decreased masticatory muscle compliance and leads to gradual muscular atrophy and degradation (40), which consequently likely leads to the buccal and temporal invagination observed in this study.

With regard to the labial region, we observed protruded lips with orthodontic treatment. The amount of protrusion was larger in the lower lips compared to the upper lips. Our findings were inconsistent with the labial retraction identified in a meta-analysis of changes of facial profile during orthodontic treatment (41). This meta-analysis investigated facial profile change before treatment and after completion of orthodontic treatment. However, our analysis focused on facial changes within the first six months. Space closure after alignment, which usually takes place after around six months of treatment, is likely to result in labial retraction that outweighs the amount of labial protrusion observed during the first six months. Debonding of brackets has been shown to result in labial retraction of $0.23 \mathrm{~mm}$ along the direction of midsagittal plane in the lower lip, which is significantly larger than the $0.04 \mathrm{~mm}$ change of the upper lip (42). It is therefore likely that the greater amount of shape change in lower lip during the first six months was due to the greater sensitivity of the lower lip towards bracket attachment. It should also be noted that patients in this study had varying degrees of anterior crowding. The initial proclination during the alignment stage may be another explanation of the observed labial protrusion. However, the relative contribution of bracket attachment and initial proclination to labial protrusion warrants further investigation. This could be achieved by standardization of the degree of anterior crowding during patient recruitment. 
The magnitude of facial changes from T0 to T2 (0.010 in unit of Procrustes distance) was smaller than the sum of the magnitude of facial changes from T0 to T1 (0.009) and from T1 to T2 (0.004). The rate of changes of facial shape from T0 to $\mathrm{T} 1$ was therefore more than twice the rate of shape change from $\mathrm{T} 1$ to T2. Likewise, the average magnitude of per-landmark change from T0 to T2 $(1.33 \mathrm{~mm})$ was smaller than the sum of average per-landmark change from T0 to T1 $(1.31 \mathrm{~mm})$ and from T1 to T2 $(0.22 \mathrm{~mm})$. Analysis of both entire set of Procrustes coordinates and per-landmark analysis therefore converge in suggesting that facial changes were concentrated within the first three months and the patterns of changes of facial shape differed between the first three months and the second three months. The relatively smaller magnitude of changes in dentition may explain decreased rate of shape change from fourth to sixth month. Different patterns of changes of facial shape from first to third month and from fourth to sixth month suggested that factors driving changes of facial shape differed during the two periods.

Few studies have investigated variance of facial shape. Among Hong Kong adolescents, variance of frontal facial shape decreased significantly among females but not among males from 12 to 18 years (15). For lateral facial images, significant reduction in variance of facial shape was observed among both genders. Our study represents the first time orthodontic treatment-related changes in variance of facial shape/form has been investigated. We observed that variance of facial shape remained relatively stable throughout alignment stage of adult orthodontic treatment. Our findings were therefore partially inconsistent with the notion of "bracket face" (9), which should lead to reduce variance of facial shape/form with ongoing orthodontic treatment. However, it remains unclear as to how variance of facial shape would change during space closure and detailing stage of orthodontic treatment.

Tooth extraction was found to have no impact on changes in mean and variance of facial shape/form. Controversies exist as to whether extraction has an impact on changes of facial shape during orthodontic treatment $(6,43)$. Well-controlled studies based on adequate sample size and advanced morphometric methods are warranted to provide further evidence in this respect.

Several limitations of the study bear noting. First, there are facial regions beyond those covered by the anthropometric mask that are of theoretical interest. More detailed changes in temporal and buccal region would be investigated if the anthropometric mask could be extended further bilaterally. However, facial regions covered by the anthropometric mask could be reconstructed with high level of fidelity. Our findings therefore provided unprecedented resolution in accurately describing facial shape changes associated with orthodontic changes among adult females. Second, the number of patients who received orthodontic treatment without tooth extraction was small in our sample because of the practical difficulty of recruiting female orthodontic patients who do not need tooth extraction. It is unclear to what extent the lack of differences in patterns of changes of facial shape between the extraction and non-extraction group is an artefact of the small number of non-extraction patients. Equal number of patients in both groups are advised for further studies. Third, our analysis focused on facial changes during the orthodontic treatment stage of tooth alignment. Longer periods of follow-ups are necessary to gain a complete understanding of facial changes throughout the entire orthodontic treatment. Fourth, the 
sample size of the present study is relatively limited. Future studies with larger sample size among populations of various ethnic backgrounds are meaningful in evaluating the robustness and generalizability of the present findings. Nevertheless, comparable sample size to earlier studies $(6,16)$ and detailed facial morphological characterization through spatially dense facial quasi-landmarks allowed the present study to be adequately powered to identify changes of facial shape.

Our findings are of clinical significance. Although the present study failed to substantiate the notion of "bracket face", buccal and temporal invagination and labial protrusion were identified as common changes among adult female orthodontic patients. These changes were concentrated in the first 3 months of treatment and seemed robust to treatment modality (tooth extraction vs non-extraction). Clinical encounter of these facial changes should not be mistaken as unexpected or treatment failure. Orthodontists are advised to be aware of these changes so as to avoid unnecessary adjustment of treatment plan. Furthermore, being cognizant of these changes will allow orthodontists to better communicate with patients so that patients will have a more realistic expectation of the process and outcome of orthodontic treatment.

\section{Conclusion}

Changes of facial shape during the first three months were twice as fast as changes from fourth to sixth month among adult females receiving orthodontic treatment. These changes were characterized by invaginated temporal and buccal regions and protruded labial region. Awareness of these facial changes will help avoid unnecessary adjustment of orthodontic treatment plan and promote communication between orthodontists and patients by helping patients developing a more realistic expectation towards orthodontic treatment.

\section{Declarations}

- Ethics approval and consent to participate

This study was approved by the Research Ethics Board of the Stomatological Hospital of Chongqing Medical University (No. 2020-013). All patients gave informed consent prior to participation.

\section{- Consent for publication}

All presentations of case reports have consent for publication.

\section{- Availability of data and materials}

The data sets generated and analyzed during the current study are not publicly available due original facial images and coordinates for vertices extracted from MeshMonk software can be used to identify study participants. But are available from the corresponding author on reasonable request.

\section{- Conflict of interest}


The authors declare no conflict of interest.

\section{- Funding}

This work was supported by the National Science Foundation for Young Scientists of China [Grant No. 31800818]; the Program for the Natural Science Foundation of Chongqing, China [cstc2018jcyjAX0220]. In addition, the work described in this paper was supported by a grant from the Research Grants Council of the Hong Kong Special Administrative Region, China [Project No. 17109619], URL: http://www.ugc.edu.hk/eng/rgc/. The funding recipient was Hai Ming Wong.

\section{- Authors' contributions}

SYH collected all patient data and was a major contributor in writing the manuscript. WWZ, HWD and JPZ was responsible for orthodontic clinical guidance. YFW and HMW extracted and analyzed the patient's facial data.

\section{- Acknowledgements}

Not Applicable.

\section{References}

1. Ferrario VF, Sforza C, Schmitz JH, Miani A Jr, Serrao G. A three-dimensional computerized mesh diagram analysis and its application in soft tissue facial morphometry. Am J Orthod Dentofacial Orthop. 1998;114(4):404-413.

2. Hay GG. Psychiatric aspects of cosmetic nasal operations. Br J Psychiatry. 1970;116(530):85-97.

3. Phillips $\mathrm{C}$, Broder HL, Bennett ME. Dentofacial disharmony: motivations for seeking treatment. Int $J$ Adult Orthodon Orthognath Surg. 1997;12(1):7-15.

4. Boley JC, Pontier JP, Smith S, Fulbright M. Facial changes in extraction and nonextraction patients. Angle Orthod. 1998;68(6):539-546.

5. James RD. A comparative study of facial profiles in extraction and nonextraction treatment. Am J Orthod Dentofacial Orthop. 1998;114(3):265-276.

6. Kouli A, Papagiannis A, Konstantoni N, Halazonetis DJ, Konstantonis D. A geometric morphometric evaluation of hard and soft tissue profile changes in borderline extraction versus non-extraction patients. Eur J Orthod. 2019;41(3):264-272.

7. Moss JP, Ismail SF, Hennessy RJ. Three-dimensional assessment of treatment outcomes on the face. Orthod Craniofac Res. 2003;6 Suppl 1:126-182.

8. Jang KS, Bayome M, Park JH, Park KH, Moon HB, Kook YA. A three-dimensional photogrammetric analysis of the facial esthetics of the Miss Korea pageant contestants. Korean J Orthod. 2017;47(2):87-99. 
9. Qin Z, Gao J, Wang J, et al. Comparative study of 3D facial photography with traditional photography[J]. Journal of Practical Stomatology, 2018, 34(2): 234-238.

10. Heil A, Lazo Gonzalez E, Hilgenfeld T, et al. Lateral cephalometric analysis for treatment planning in orthodontics based on MRI compared with radiographs: A feasibility study in children and adolescents. PLoS One. 2017;12(3).

11. Slice D E. Geometric morphometrics[J]. Annual Review of Anthropology, 2007, 36.

12. Modern morphometrics in physical anthropology[M]. Springer Science \& Business Media, 2006.

13. Dryden I L, Mardia K V. Statistical shape analysis: with applications in R[M]. John Wiley \& Sons, 2016.

14. Archaeological science: an introduction[M]. Cambridge University Press, 2020.

15. Wen YF, Wong HM, McGrath CP. Developmental shape changes in facial morphology: Geometric morphometric analyses based on a prospective, population-based, Chinese cohort in Hong Kong. PLoS One. 2019;14(6):e0218542. Published 2019 Jun 28.

16. Abedini S, Elkenawy I, Kim E, Moon W. Three-dimensional soft tissue analysis of the face following micro-implant-supported maxillary skeletal expansion. Prog Orthod. 2018;19(1):46. Published 2018 Nov 19.

17. Neubauer S, Gunz P, Scott NA, Hublin JJ, Mitteroecker P. Evolution of brain lateralization: A shared hominid pattern of endocranial asymmetry is much more variable in humans than in great apes. Sci Adv. 2020;6(7):eaax9935. Published 2020 Feb 14.

18. https://www.cdc.gov/healthyweight/assessing/bmi/adult_bmi/index.html

19. Heike CL, Upson K, Stuhaug E, Weinberg SM. 3D digital stereophotogrammetry: a practical guide to facial image acquisition. Head Face Med. 2010;6:18. Published 2010 Jul 28.

20. Wiley D F, Amenta N, Alcantara D A, et al. Evolutionary morphing[M]. IEEE, 2005.

21. White JD, Ortega-Castrillón A, Matthews $H$, et al. MeshMonk: Open-source large-scale intensive 3D phenotyping. Sci Rep. 2019;9(1):6085. Published 2019 Apr 15.

22. Claes $\mathrm{P}$, Walters $\mathrm{M}$, Clement J. Improved facial outcome assessment using a 3D anthropometric mask. Int J Oral Maxillofac Surg. 2012;41(3):324-330.

23. Mardia K V, Bookstein F L, Moreton I J. Statistical assessment of bilateral symmetry of shapes[J]. Biometrika, 2000: 285-300.

24. Rohlf F J, Slice D. Extensions of the Procrustes method for the optimal superimposition of landmarks[J]. Systematic Biology, 1990, 39(1): 40-59.

25. McArdle B H, Anderson M J. Fitting multivariate models to community data: a comment on distancebased redundancy analysis[J]. Ecology, 2001, 82(1): 290-297.

26. Anderson M, Braak C T. Permutation tests for multi-factorial analysis of variance[J]. Journal of statistical computation and simulation, 2003, 73(2): 85-113.

27. Kleinbaum D G, Kupper L L, Nizam A, et al. Applied regression analysis and other multivariable methods[M]. Nelson Education, 2013. 
28. Zaidi AA, White JD, Mattern BC, et al. Facial masculinity does not appear to be a condition-dependent male ornament and does not reflect MHC heterozygosity in humans. Proc Natl Acad Sci U SA. 2019;116(5):1633-1638.

29. Claes $P$, Walters $M$, Shriver MD, et al. Sexual dimorphism in multiple aspects of 3D facial symmetry and asymmetry defined by spatially dense geometric morphometrics. J Anat. 2012;221(2):97-114.

30. Oksanen J, Blanchet F G, Kindt R, et al. Package 'vegan'[J]. Community ecology package, version, 2013, 2(9): 1-295.

31. Vegan package: Community Ecology Package. R package version5-6. https://CRAN.Rproject.org/package=vegan

32. Foote M. Contributions of individual taxa to overall morphological disparity[J]. Paleobiology, 1993: 403-419.

33. Adams D C, Otárola-Castillo E. geomorph: an R package for the collection and analysis of geometric morphometric shape data[J]. Methods in Ecology and Evolution, 2013, 4(4): 393-399.

34. Adams, D. C., M. L. Collyer, and A. Kaliontzopoulou. 2020. Geomorph: Software for geometric morphometric analyses. $R$ package version 3.2.1 . http://cran.r-project.org/package=geomorph.

35. Wirthlin J, Kau CH, English JD, Pan F, Zhou H. Comparison of facial morphologies between adult Chinese and Houstonian Caucasian populations using three-dimensional imaging. Int J Oral Maxillofac Surg. 2013;42(9):1100-1107.

36. Takema Y, Tsukahara K, Fujimura T, Hattori M. Age-related changes in the three-dimensional morphological structure of human facial skin. Skin Res Technol. 1997;3(2):95-100.

37. Otasevic M, Naini FB, Gill DS, Lee RT. Prospective randomized clinical trial comparing the effects of a masticatory bite wafer and avoidance of hard food on pain associated with initial orthodontic tooth movement. Am J Orthod Dentofacial Orthop. 2006;130(1):.

38. LIANG C, HUANG C. Comparison study on teenager'masticatory efficiency before and after extraction orthodontics therapy[J]. China Journal of Modern Medicine, 2011 (11): 32.

39. Farella M, Michelotti A, Carbone G, Gallo LM, Palla S, Martina R. Habitual daily masseter activity of subjects with different vertical craniofacial morphology. Eur J Oral Sci. 2005;113(5):380-385.

40. Bosse J P, Papillon J. Surgical anatomy of the SMAS at the malar region[C]//Transactions of the 9th International Congress of Plastic and Reconstructive Surgery. New York: McGraw-Hill. 1987, $348 .$.

41. Konstantonis D, Vasileiou D, Papageorgiou SN, Eliades T. Soft tissue changes following extraction vs. nonextraction orthodontic fixed appliance treatment: a systematic review and meta-analysis. Eur J Oral Sci. 2018;126(3):167-179.

42. Kim YK, Lee NK, Moon SW, Jang MJ, Kim HS, Yun PY. Evaluation of soft tissue changes around the lips after bracket debonding using three-dimensional stereophotogrammetry. Angle Orthod. 2015;85(5):833-840.

43. Ismail SF, Moss JP, Hennessy R. Three-dimensional assessment of the effects of extraction and nonextraction orthodontic treatment on the face. Am J Orthod Dentofacial Orthop. 2002;121(3):244- 


\section{Tables}

Table 1. Main effect of treatment stage, tooth extraction group, and their interaction from permutational MANOVA

\begin{tabular}{lcclcc}
\hline & \multicolumn{2}{c}{ Facial shape } & & \multicolumn{2}{c}{ Facial form } \\
\cline { 2 - 3 } \cline { 5 - 6 } & partial $\mathrm{R}^{2}$ & $\mathrm{p}$-value & & partial $\mathrm{R}^{2}$ & $\mathrm{p}$-value \\
\hline Treatment stage & 0.00929 & $0.0030^{* *}$ & & 0.01551 & 0.1030 \\
\hline Extraction Group & 0.06100 & $0.0062^{* *}$ & & 0.11565 & 0.1277 \\
\hline $\begin{array}{l}\text { Treatment stage } \\
\text { Extraction Group }\end{array}$ & 0.00221 & 0.3437 & & 0.00294 & 0.3209 \\
\hline
\end{tabular}

$* * \mathrm{p} \square 0.01$

Partial $\mathrm{R}^{2}=$ coefficient of partial determination

Table 2. Pairwise differences in mean shape and form among treatment stages

\begin{tabular}{|c|c|c|c|c|c|}
\hline & \multicolumn{3}{|c|}{ Facial shape } & \multicolumn{2}{|c|}{ Facial form } \\
\hline & partial $\mathrm{R}^{2}$ & $p$-value & & partial $\mathrm{R}^{2}$ & p-value \\
\hline T0 T1 & 0.011 & 0.0059 & ** & 0.02113 & 0.0759 \\
\hline $\mathrm{T} 1 \sim \mathrm{T} 2$ & 0.0026 & 0.4728 & & 0.00142 & 0.6517 \\
\hline T0 T2 & 0.0151 & 0.0098 & ** & 0.02223 & 0.0547 \\
\hline
\end{tabular}

$* * p \square 0.01$

Partial $\mathrm{R}^{2}=$ coefficient of partial determination

Table 3. Pairwise changes in variance of facial shape and form in extraction and nonextraction group

\begin{tabular}{cccccc}
\hline & \multicolumn{2}{c}{ Extraction group } & & \multicolumn{2}{c}{ Non-extraction group } \\
\cline { 2 - 3 } \cline { 5 - 6 } & $\Delta$ Var & $\mathrm{p}$-values & & $\Delta$ Var & p-values \\
\hline Shape variance & & & & \\
T0-T1 & 0.000340539 & 0.3835 & & 0.000164982 & 0.8178 \\
T1-T2 & 0.000174259 & 0.6566 & & 0.000012994 & 0.9847 \\
T0-T2 & 0.000514798 & 0.1844 & & 0.000177976 & 0.8818 \\
\hline Form variance & & & & \\
T0-T1 & 195196.8 & 0.3077 & & 3064.043 & 0.9264 \\
T1-T2 & 156.5571 & 0.9959 & & 394.0572 & 0.9922 \\
T0-T2 & 195353.3 & 0.3174 & 2669.986 & 0.9368 \\
\hline
\end{tabular}


$\Delta$ Var: difference in Procrustes variance of facial shape/form between the two time periods under comparison.

\section{Figures}

A

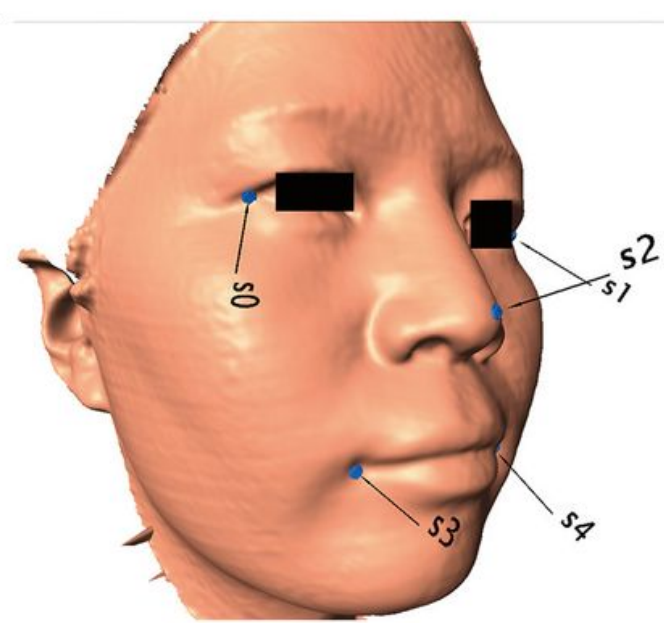

B

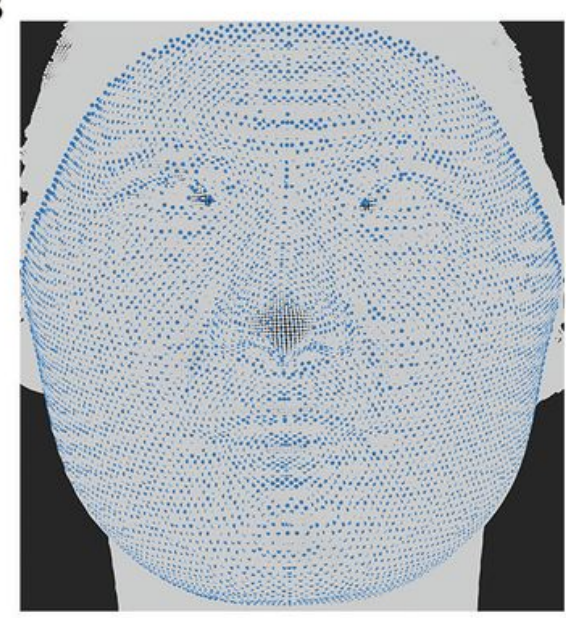

$\mathrm{C}$

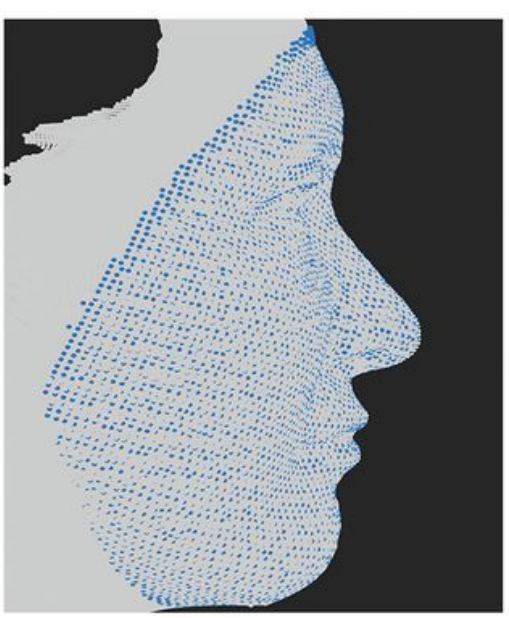

Figure 1

Demonstration of facial mapping. (A). Digitization of five anchoring landmarks. Spatially dense facial landmarks are mapped onto target face as shown in (B) frontal and (C) lateral view.

A

B
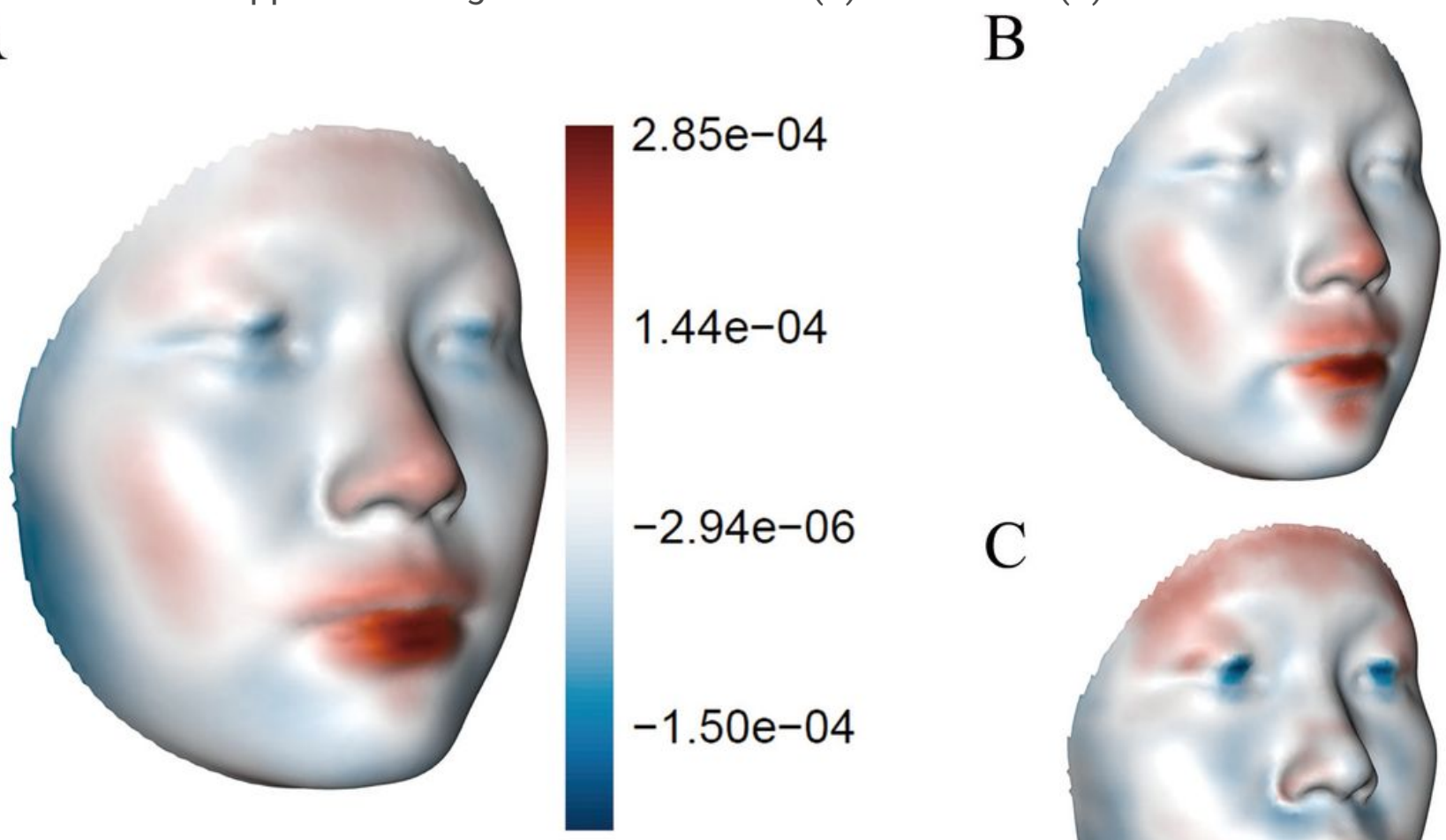

$1.35 e+00$

$6.84 e-01$

$-1.40 e-02$

$-7.12 e-01$

C
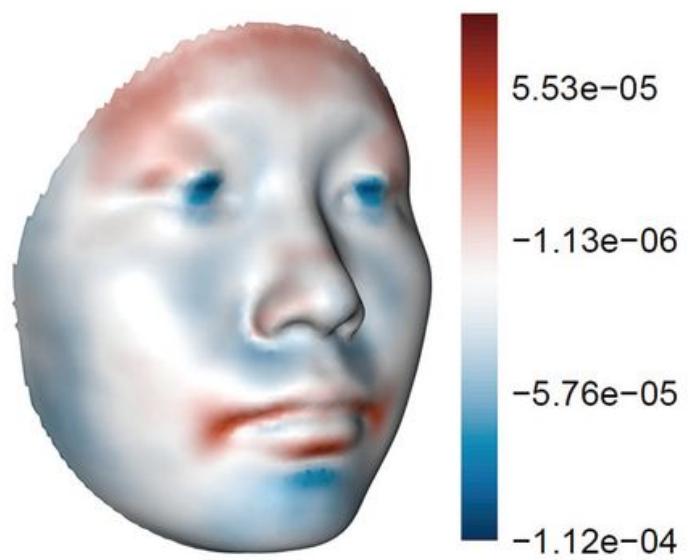

Figure 2 
Changes of facial shape during the first six months of orthodontic treatment. Changes of facial shape are illustrated. (A). T0 to T2; (B). T0 to T1; (C). T1 to T2.

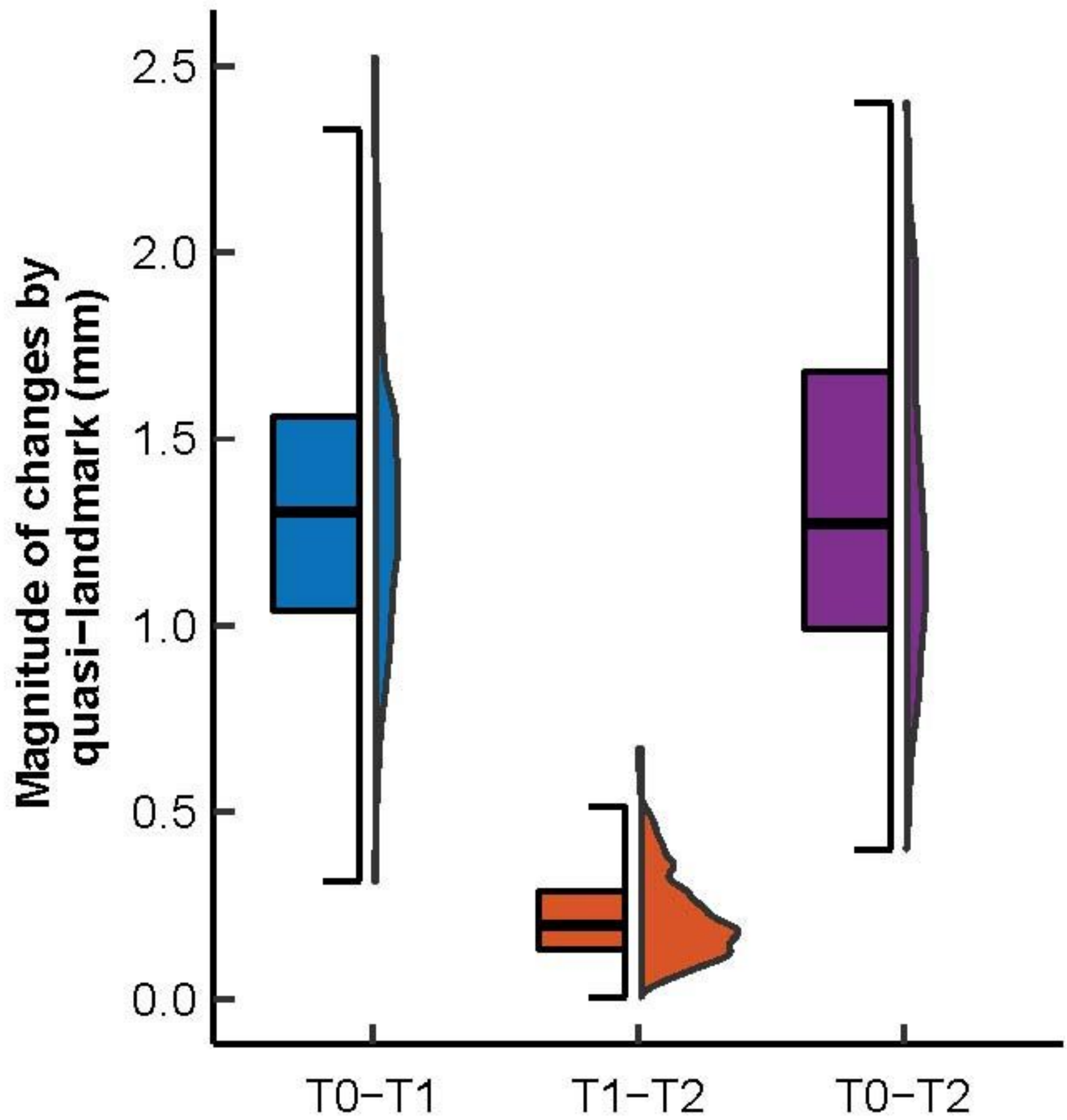

Figure 3

Box plot of distribution of magnitude of changes of quasi-landmarks by treatment stage. (A). T0 to T2; (B). T0 to T1; (C). T1 to T2. Horizontal bar inside box plot indicates the median value. Distribution of magnitude of changes were plotted in histogram next to the box plot. 
A

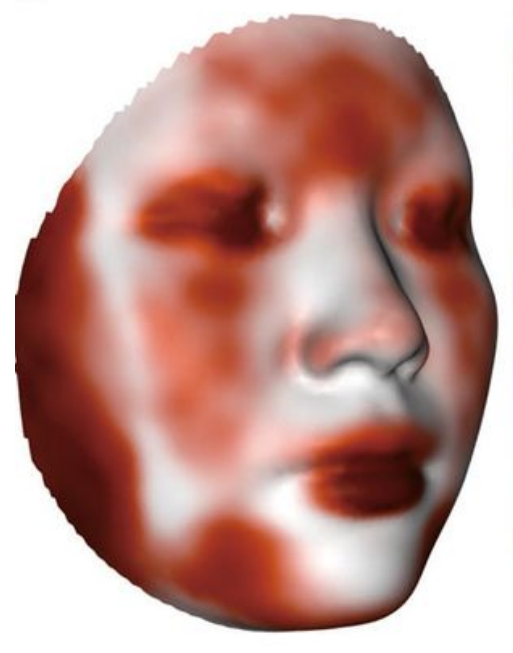

$3.43 e-02$

$2.74 e-02$

$2.06 e-02$

$1.37 e-02$

$6.87 e-03$

$2.27 e-05$
B

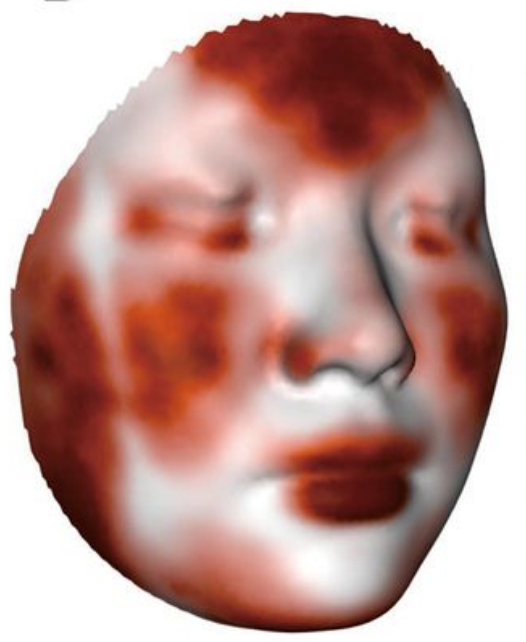

C

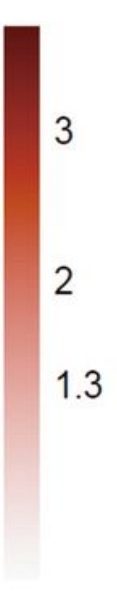

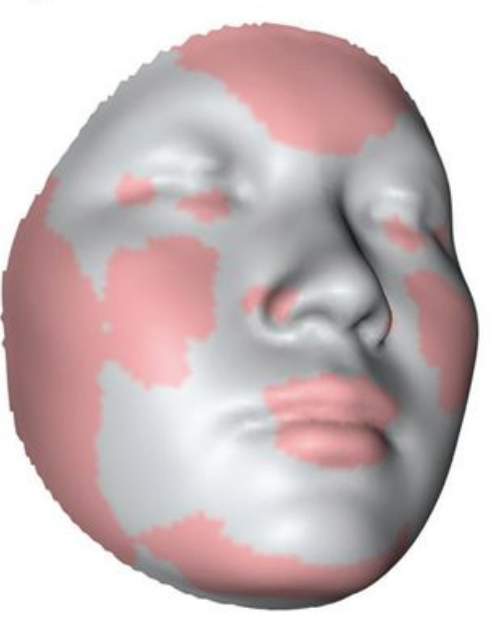

Figure 4

Overall changes of facial shape within the first six months. Heatmap illustrating (A) $R^{\wedge} 2$ and $(B)$ negative log base 10 of the $p$ value. In figure (C), facial regions with significant changes were marked in pink. 